\title{
Fault Diagnosis for Linear Discrete Systems Based on an Adaptive Observer
}

\author{
Jianwei Liu, Bin Jiang, and Ke Zhang \\ College of Automation Engineering, Nanjing University of Aeronautics and Astronautics, Nanjing 210016, China \\ Correspondence should be addressed to Bin Jiang; ebjiang@yahoo.com
}

Received 9 July 2013; Revised 9 September 2013; Accepted 23 September 2013

Academic Editor: Zidong Wang

Copyright (C) 2013 Jianwei Liu et al. This is an open access article distributed under the Creative Commons Attribution License, which permits unrestricted use, distribution, and reproduction in any medium, provided the original work is properly cited.

\begin{abstract}
This paper presents a fault diagnosis algorithm to estimate the fault for a class of linear discrete systems based on an adaptive fault estimation observer. And observer gain matrix and adaptive adjusting rule of the fault estimator are designed. Furthermore, the adaptive regulating algorithm can guarantee the first-order difference of a Lyapunov discrete function to be negative, so that the observer is ensured to be stable and fault estimation errors are convergent. Finally, simulation results of an aircraft F-16 illustrate the advantages of the theoretic results that are obtained in this paper.
\end{abstract}

\section{Introduction}

As the scale and complexity of modern control systems are increasing, the requirements on system reliability are also increasing. Therefore, the design and analysis of fault detection and diagnosis (FDD) algorithms have received considerable attention during the past three decades. The development of FDD has been addressed by more and more authors and fruitful results have been obtained; see [1-3].

The observer technology is one of the important methods for FDD and fault-tolerant control $[4,5]$. Commonly used observer-based fault estimation methods include sliding mode observers [6], unknown input observers [7], $H_{\infty}$ filtering methods [8], neural networks observers [9], and adaptive observers [10,11]. But most of them focus on the continuous systems, and only few results have been reported on the fault estimation design in discrete-time systems. The discrete-time is widely used in practical implementations, for example, computer control systems, networked control systems, and so forth [12]. Reference [13] designed an $H_{\infty}$ FD filter for a class of linear discrete-time systems in a networked environment. Reference [14] used an adaptive fault observer to deal with discrete-time systems, but it did not involve the issue of fault estimation. Reference [15] also dealt with discrete-time nonlinear systems, but its inequality functions were complex and it was difficult to get solutions. On the other hand, aircraft flight control systems are good examples for applications of fault accommodation/active reliable control, and the fault observers have been employed to detect faults [11].

In this paper, a novel discrete-time actuator fault estimation scheme is proposed to deal with abrupt actuator failures. The proposed actuator fault estimation scheme is then applied by using the Lyapunov method. Simulation results of a numerical example are also given.

The paper is organized as follows. Section 2 describes the mathematical preliminaries and problem formulation. In Section 3, concerning the theoretical results of the proposed fault diagnosis scheme, a fault estimation scheme is proposed to deal with the actuator failures of discrete-time system. In Section 4, an example of aircraft flight control system is given to illustrate the performance of the proposed scheme. The concluding remarks are given in Section 5.

\section{System Description and Preliminaries}

Consider a discrete-time linear system

$$
\begin{gathered}
x(k+1)=A x(k)+B u(k)+E f(k), \\
y(k)=C x(k),
\end{gathered}
$$

where $x(k) \in R^{n}$ is the state, $u \in R^{m}$ is the control input, $f(k) \in R^{q}$ with $q \leq m$ is the function to model the actuator faults, and $y(k) \in R^{r}$ is the measurable output. Matrices $A, B$, 
$C$, and $E$ are real matrices of appropriate dimensions. Matrix $E$ is of full column rank; that is, $\operatorname{rank}(E)=q$.

For estimating the actuator fault $f(k)$, an adaptive observer is constructed as follows:

$$
\begin{gathered}
\widehat{x}(k+1)=A \hat{x}(k)+B u(k)+E \widehat{f}(k)-L[\widehat{y}(k)-y(k)], \\
\widehat{y}(k)=C \widehat{x}(k),
\end{gathered}
$$

where $\widehat{x}(k) \in R^{n}$ is the observer state vector, $\widehat{y}(k) \in R^{r}$ is the observer output vector, $\widehat{f}(k) \in R^{q}$ is the estimate of the actuator fault $f(k), L \in R^{n \times r}$ is the observer gain to be designed.

Denoting that

$$
\begin{gathered}
e_{x}=\widehat{x}(k)-x(k), \quad e_{y}=\widehat{y}(k)-y(k), \\
e_{f}=\widehat{f}(k)-f(k),
\end{gathered}
$$

then the estimation error dynamics is modeled as follows:

$$
\begin{gathered}
e_{x}(k+1)=(A-L C) e_{x}(k)+E e_{f}(k), \\
e_{y}(k)=C e_{x}(k)
\end{gathered}
$$

\section{Main Results}

\subsection{Modified Adaptive Fault Estimation Algorithm}

Theorem 1. For constant actuator fault $f(k)$, if there exist matrices $P>0$ and $Q>0$ and positive scalars $\varepsilon$ and defined

$$
\Gamma=2\left(\varepsilon E E^{T}+Q\right)^{-1}
$$

such that the following condition holds:

$$
\Xi=\left[\begin{array}{cc}
(A-L C)^{T} P(A-L C)-P & (A-L C)^{T} P E \\
* & E^{T}\left(-\Gamma^{T} Q \Gamma+P\right) E
\end{array}\right]<0,
$$

then the algorithm

$$
\Delta \widehat{f}(k+1)=-\varepsilon E^{T} \Gamma\left[e_{x}(k+1)-(A-L C) e_{x}(k)\right]
$$

and the fault estimation

$$
\widehat{f}(k+1)=\widehat{f}(k)+\Delta \widehat{f}(k+1)=\widehat{f}(0)+\sum_{i=1}^{k+1} \Delta \widehat{f}(i)
$$

can realize estimation error of both the state and fault uniformly bounded for the entire time period.

Proof. From system (4), one gets $E e_{f}(k)=e_{x}(k+1)-(A-$ $L C) e_{x}(k)$, so the algorithm (7) becomes

$$
\begin{aligned}
\Delta \widehat{f}(k+1) & =-\varepsilon E^{T} \Gamma\left[e_{x}(k+1)-(A-L C) e_{x}(k)\right] \\
& =-\varepsilon E^{T} \Gamma E e_{f}(k)
\end{aligned}
$$

Then,

$$
\begin{aligned}
e_{f}(k+1) & =\widehat{f}(k+1)-f(k+1) \\
& =\widehat{f}(k+1)-\widehat{f}(k)+\widehat{f}(k)-f(k+1) .
\end{aligned}
$$

Because $f(k)$ is constant, $f(k+1)=f(k)$ and $\Delta \widehat{f}(k+1)=$ $\widehat{f}(k+1)-\widehat{f}(k)$; then one gets

$$
e_{f}(k+1)=\Delta \widehat{f}(k+1)+e_{f}(k)=\left(I-\varepsilon E^{T} \Gamma E\right) e_{f}(k) .
$$

Based on (4) and (11), we can obtain the following augmented system:

$$
\left[\begin{array}{l}
e_{x}(k+1) \\
e_{f}(k+1)
\end{array}\right]=\left[\begin{array}{cc}
A-L C & E \\
0 & I-\varepsilon E^{T} \Gamma E
\end{array}\right]\left[\begin{array}{l}
e_{x}(k) \\
e_{f}(k)
\end{array}\right] .
$$

Consider the following Lyapunov function:

$$
V(k)=e_{x}^{T}(k) P e_{x}(k)+\varepsilon^{-1} e_{f}^{T}(k) e_{f}(k) .
$$

Then,

$$
\begin{aligned}
\Delta V(k)= & e_{x}^{T}(k+1) P e_{x}(k+1)-e_{x}^{T}(k) P e_{x}(k) \\
& +\varepsilon^{-1} e_{f}^{T}(k+1) e_{f}(k+1)-\varepsilon^{-1} e_{f}^{T}(k) e_{f}(k) \\
= & e_{x}^{T}(k)\left[(A-L C)^{T} P(A-L C)-P\right] e_{x}(k) \\
& +2 e_{x}^{T}(k)(A-L C)^{T} P E e_{f}(k)+e_{f}^{T}(k) E^{T} P E e_{f}(k) \\
& +\varepsilon^{-1} e_{f}^{T}(k)\left(I-E^{T} \Gamma E\right)^{T}\left(I-E^{T} \Gamma E\right) e_{f}(k) \\
& -\varepsilon^{-1} e_{f}^{T}(k) e_{f}(k) \\
= & e_{x}^{T}(k)\left[(A-L C)^{T} P(A-L C)-P\right] e_{x}(k) \\
& +2 e_{x}^{T}(k)(A-L C)^{T} P E e_{f}(k) \\
& +e_{f}^{T}(k) E^{T}\left(-\Gamma^{T} Q \Gamma+P\right) E e_{f}(k) .
\end{aligned}
$$

Denoting that $e(k)=\left[e_{x}(k) e_{f}(k)\right]^{T}$, then $\Delta V(k)=$ $e(k)^{T} \Xi e(k)<0$, and $\Xi$ is defined as (6).

Remark 2. The pair $(A, C)$ is observable, and define the observe matrix $U=\left[C^{T}(C A)^{T} \ldots\left(C A^{l-1}\right)^{T}\right]^{T}$. So, it gets that the rank of $U$ is $n$. When $C$ is full column rank,we have

$$
\begin{aligned}
\Delta \widehat{f}(k+1) & =-\varepsilon E^{T} \Gamma\left[e_{x}(k+1)-(A-L C) e_{x}(k)\right] \\
& =-\varepsilon E^{T} \Gamma\left[C^{-1} e_{y}(k+1)-\left(A C^{-1}-L\right) e_{y}(k)\right] .
\end{aligned}
$$

At the first step, the online fault estimation is as follows:

$$
\begin{aligned}
\widehat{f}(1) & =\widehat{f}(0)+\Delta \widehat{f}(1) \\
& =\widehat{f}(0)-\varepsilon E^{T} \Gamma\left[C^{-1} e_{y}(1)-\left(A C^{-1}-L\right) e_{y}(0)\right] .
\end{aligned}
$$

And at the $k$ th step, fault estimation is as (8). 
If $C$ is not full column rank, then more system outputs and observer estimations should be used to get the state estimation.

When the output $y(k)$ satisfies Lipschitz condition, define $l_{\min }=\operatorname{argmax}_{l}[\operatorname{rank}(U)]$, and define $F=(A-L C)^{-1}$ and $U_{F}=\left[C^{T}(C F)^{T} \cdots\left(C F^{l \min -1}\right)^{T}\right]^{T}$; then one can get

$$
\begin{aligned}
\Delta \widehat{f}(k+1) & \\
=-\varepsilon E^{T} \Gamma & \left\{U_{F}^{-1}\left[\begin{array}{lll}
e_{y}(k+1)^{T} & \cdots & e_{y}(k+2-l \mathrm{~min})^{T}
\end{array}\right]^{T}\right. \\
& -A U_{F}^{-1}\left[\begin{array}{lll}
e_{y}(k)^{T} & \cdots & e_{y}(k+1-l \mathrm{~min})^{T}
\end{array}\right] \\
& \left.+L e_{y}(k)\right\} .
\end{aligned}
$$

Then, fault estimation is $\widehat{f}(k+1)=\widehat{f}(k)+\Delta \widehat{f}(k+1)=\widehat{f}(0)+$ $\sum_{i=1}^{k+1} \Delta \widehat{f}(i)$.

Remark 3. Equation (6) is a nonlinear matrix inequality about matrices $\Gamma$ and $Q$, and it is not easy to be calculated, so let $Q=\varepsilon E E^{T}+\delta \operatorname{tr}\left(E E^{T}\right)$. And after we choose $0<\delta<\varepsilon$, the solution can be found easily.

Remark 4. If the fault $f(k)$ is not constant but is a linear function, such as $f(k+1)=H f(k)$, then

$$
e_{f}(k+1)=\left(H-E^{T} \Gamma E\right) e_{f}(k) .
$$

Consider the same Lyapunov function $V(k)=e_{x}^{T}(k) P e_{x}(k)+$ $\varepsilon^{-1} e_{f}^{T}(k) e_{f}(k)$. We can also get

$$
\Xi=\left[\begin{array}{cc}
(A-L C)^{T} P(A-L C)-P & (A-L C)^{T} P E \\
* & E^{T}\left(-\varepsilon \Gamma^{T} Q \Gamma+P\right) E+\varepsilon\left(H^{T} H-I\right)
\end{array}\right]<0 .
$$

The proof is smilar to that of Theorem 1 and it is omitted here for brevity.

\section{Simulation Results}

In this section, the fault estimation algorithm is applied to a model of the vertical dynamics of an F-16 aircraft. The model is taken from [16]. The signals and their generation in the simulations are summarized in Table 1, where size means the variance for the inputs and constant magnitude for the faults, respectively.
TABLE 1: Signals in the F-16 simulation study.

\begin{tabular}{lclc}
\hline Signal & Not. & Meaning & Size \\
\hline \multirow{4}{*}{ Inputs } & $u_{1}$ & Spoiler angle $(0.1 \mathrm{deg})$ & 1 \\
& $u_{2}$ & Forward accelerations $\left(\mathrm{m} / \mathrm{s}^{2}\right)$ & 1 \\
& $u_{3}$ & Elevator angle $(\mathrm{deg})$ & 1 \\
\hline \multirow{4}{*}{ Outputs } & $y_{1}$ & Relative altitude $(\mathrm{m})$ & $10^{-4}$ \\
& $y_{2}$ & Forward speed $(\mathrm{m} / \mathrm{s})$ & $10^{-6}$ \\
& $y_{3}$ & Pitch angle $(\mathrm{deg})$ & $10^{-6}$ \\
\hline \multirow{4}{*}{ States } & $x_{1}$ & Altitude $(\mathrm{m})$ & $10^{-4}$ \\
& $x_{2}$ & Forward speed $(\mathrm{m} / \mathrm{s})$ & $10^{-4}$ \\
& $x_{3}$ & Pitch angle $(\mathrm{deg})$ & $10^{-4}$ \\
& $x_{4}$ & Pitch rate $(\mathrm{deg} / \mathrm{s})$ & $10^{-4}$ \\
& $x_{5}$ & Vertical speed $(\mathrm{deg} / \mathrm{s})$ & $10^{-4}$ \\
\hline \multirow{4}{*}{ Faults } & $f_{1}$ & Spoiler angle actuator & 0.5 \\
& $f_{2}$ & Forward acceleration actuator & 0.1 \\
& $f_{3}$ & Elevator angle actuator & 1 \\
\hline
\end{tabular}

We have the following numerical values in (1):

$$
\begin{aligned}
& A=\left[\begin{array}{ccccc}
1 & 0.0014 & 0.1133 & 0.0004 & -0.0997 \\
0 & 0.9945 & -0.0171 & -0.0005 & 0.0070 \\
0 & 0.0003 & 1 & 0.0957 & -0.0049 \\
0 & 0.0061 & 0 & 0.9130 & -0.0966 \\
0 & -0.0286 & 0.0002 & 0.1004 & 0.9879
\end{array}\right], \\
& B=\left[\begin{array}{ccc}
-0.0078 & 0 & 0.0003 \\
-0.0115 & 0.0997 & 0 \\
0.0212 & 0 & -0.0081 \\
0.4150 & 0.0003 & -0.1589 \\
0.1794 & -0.0014 & -0.0158
\end{array}\right], \\
& E=\left[\begin{array}{ccc}
-0.0078 & 0 & 0.0003 \\
-0.0115 & 0.0997 & 0 \\
0.0212 & 0 & -0.081 \\
0.4150 & 0.0003 & -0.1589 \\
0.1794 & -0.0014 & -0.0158
\end{array}\right] \text {, } \\
& C=\left[\begin{array}{lllll}
1 & 0 & 0 & 0 & 0 \\
0 & 1 & 0 & 0 & 0 \\
0 & 0 & 1 & 0 & 0
\end{array}\right], \\
& l_{\min }=2, \quad U_{F}=\left[\begin{array}{ll}
C^{T} & (C F)^{T}
\end{array}\right]^{T} .
\end{aligned}
$$

By solving conditions in Theorem 1 , one can obtain the following solutions after iterations: $L=[0.000100 ; 00.0001$ $0 ; 00$ 0.0002; $0.00010 .00010 ; 00.00010 .0001] ; \varepsilon=1, \delta=$ 0.1 . Then, one can take the learning rate $\Gamma=[189923-147.6$ $646.224431727 .8 ;-147.6100 .6 \quad 1.31-0.581 .24 ; 646.21 .31$ $9365-465.8-1.27 ; 2443-0.589-465.871 .3-5.26 ; 1727.8$ $1.24-1.27-5.2687 .5]$. 


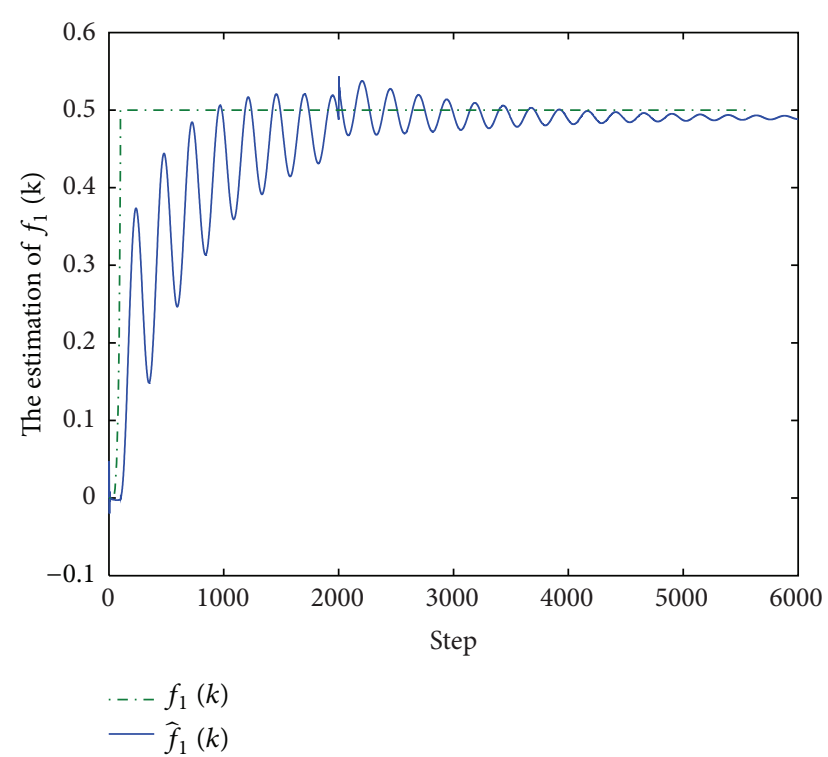

FIgURE 1: Estimation of the fault $f_{1}(k)$.

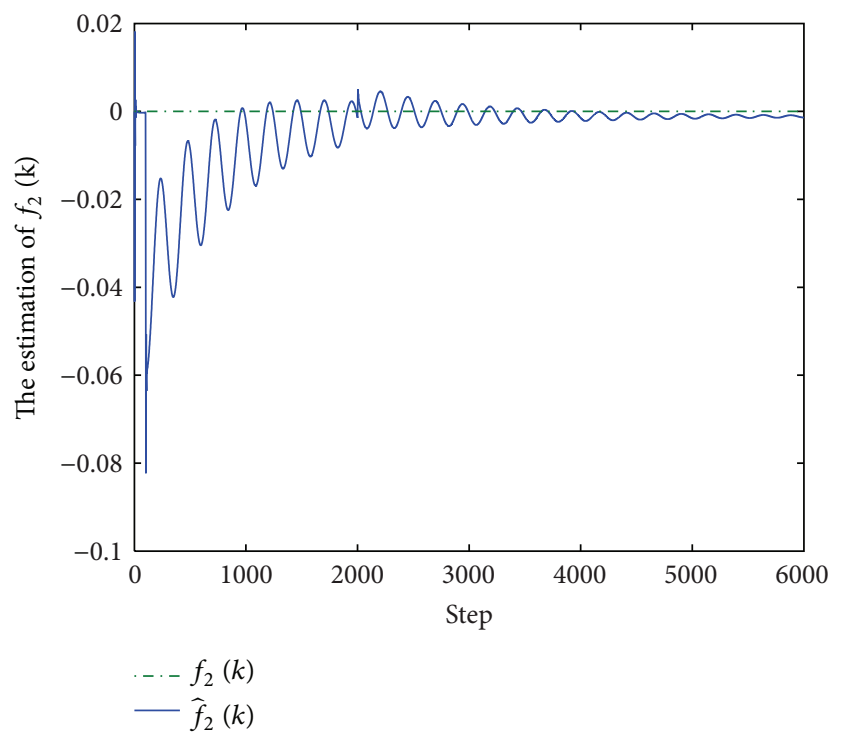

FIGURE 2: Estimation of the fault $f_{2}(k)$.

In this simulation, it is assumed that three kinds of actuator faults $f_{i}(k)$ are, respectively, created as

$$
\begin{gathered}
f_{1}(k)=\left\{\begin{array}{ll}
0, & k<100, \\
0.5, & k>100,
\end{array} f_{2}(k)\right. \text { is free, } \\
f_{3}(k)=\left\{\begin{array}{ll}
0, & k<2000 \\
1, & k>2000
\end{array} .\right.
\end{gathered}
$$

The fault $f_{1}(k)$ estimation result is shown in Figure 1, while Figure 2 illustrates the estimation of $f_{2}(k)$, and Figure 3 illustrates the estimation of $f_{3}(k)$.

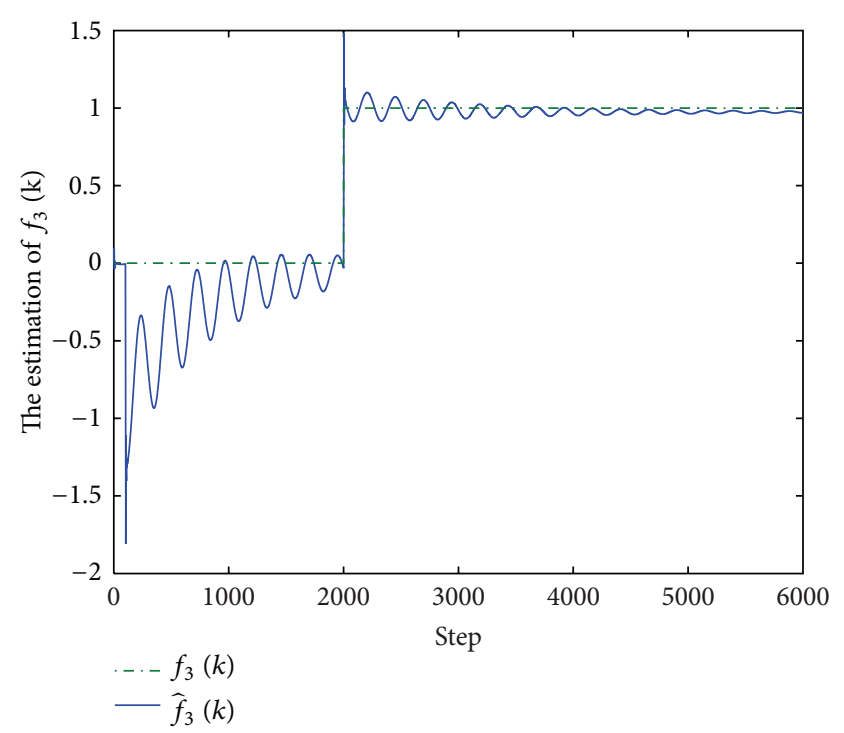

FIgURE 3: Estimation of the fault $f_{3}(k)$.

\section{Conclusions}

In this paper, a fault estimation algorithm is established for linear discrete systems with actuator faults. The algorithm can enhance the performance of fault estimation. And simulation results show that using the algorithm, the accuracy of fault estimation can be improved evidently. Extension of the proposed fault estimation method to more general nonlinear systems is an interesting issue, which will be investigated in our future research work.

\section{Acknowledgments}

This work is supported by the National Natural Science Foundation of China (nos. 61273171, 61304112, and 61104020), the Natural Science Foundation of Jiangsu Province (BK20131364), and the Doctoral Fund of Ministry of Education of China (no. 20113218110011).

\section{References}

[1] R. J. Patton, P. Frank, and R. Clark, Fault Diagnosis in Dynamic Systems: Theory and Application, Prentice-Hall, Upper Saddle River, NJ, USA, 1989.

[2] J. Chen and R. J. Patton, Robust Model-Based Fault Diagnosis for Dynamic Systems, Kluwer Academic, London, UK, 1999.

[3] B. Jiang, Z. H. Mao, H. Yang, and Y. Zhang, Fault Diagnosis and Fault Accommodation for Control Systems, National Defence Industry Press, Beijing, China, 2009.

[4] R. Marino and P. Tomei, "Adaptive observers for a class of multioutput non-linear systems," International Journal of Adaptive Control and Signal Processing, vol. 6, no. 4, pp. 353-365, 1992.

[5] D. Zhou, H. Ye, G. Wang, and X. Ding, "Discussion of some important issues of observer based fault diagnosis technique," Acta Automatica Sinica, vol. 24, no. 3, pp. 338-344, 1998.

[6] C.-F. Zhang, M. Yan, J. He, and C. Luo, "LMI-based sliding mode observers for incipient faults detection in nonlinear 
system," Journal of Applied Mathematics, vol. 2012, Article ID 528932, 13 pages, 2012.

[7] M. Witczak, J. Korbicz, and R. I. Józefowicz, "Design of unknown input observers for non-linear stochastic systems and their application to robust fault diagnosis," Control and Cybernetics, vol. 42, no. 1, pp. 227-256, 2013.

[8] Y. C. Zhang, L. N. Wu, and Z. F. Wang, "An LMI approach to mixed $H_{-} / H_{\infty}$ robust fault detection observer design," Advanced Materials Research, vol. 546, pp. 874-879, 2012.

[9] Q. Wu and M. Saif, "Robust fault detection and diagnosis in a class of nonlinear systems using a neural sliding mode observer," International Journal of Systems Science, vol. 38, no. 11, pp. 881-899, 2007.

[10] Y. Zhang and Z. Zheng, "Adaptive observer-based integrated fault diagnosis and fault-tolerant control systems against actuator faults and saturation," Journal of Dynamic Systems, Measurement, and Control, vol. 135, no. 4, Article ID 041008, pp. 1-13, 2013.

[11] B. Jiang, J. L. Wang, and Y. C. Soh, "An adaptive technique for robust diagnosis of faults with independent effects on system outputs," International Journal of Control, vol. 75, no. 11, pp. 792802, 2002.

[12] Z. Wang, H. Guan, and C. Zheng, "Fault diagnosis observer design for discrete-time delayed complex interconnected networks with linear coupling," Mathematical Problems in Engineering, vol. 2012, Article ID 860489, 22 pages, 2012.

[13] X. He, Z. Wang, Y. D. Ji, and D. Zhou, "Fault detection for discrete-time systems in a networked environment," International Journal of Systems Science, vol. 41, no. 8, pp. 937-945, 2010.

[14] F. Caccavale and L. Villani, "An adaptive observer for fault diagnosis in nonlinear discrete-time systems," in Proceedings of the American Control Conference (AAC '04), pp. 2463-2468, Boston, Mass, USA, July 2004.

[15] K. Zhang, B. Jiang, and P. Shi, "Observer-based integrated robust fault estimation and accommodation design for discretetime systems," International Journal of Control, vol. 83, no. 6, pp. 1167-1181, 2010.

[16] A. Hagenblad, F. Gustafsson, and I. Klein, "A comparison of two methods for stochastic fault detection: the parity space approach and principal component analysis," in Proceedings of the 13th IFAC Symposium on System Identification, Rotterdam, The Netherlands, August 2003. 


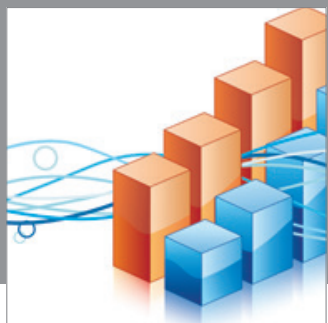

Advances in

Operations Research

mansans

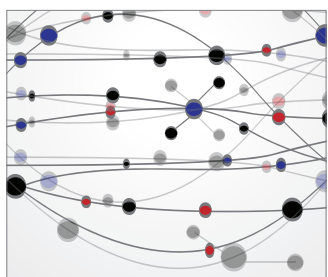

The Scientific World Journal
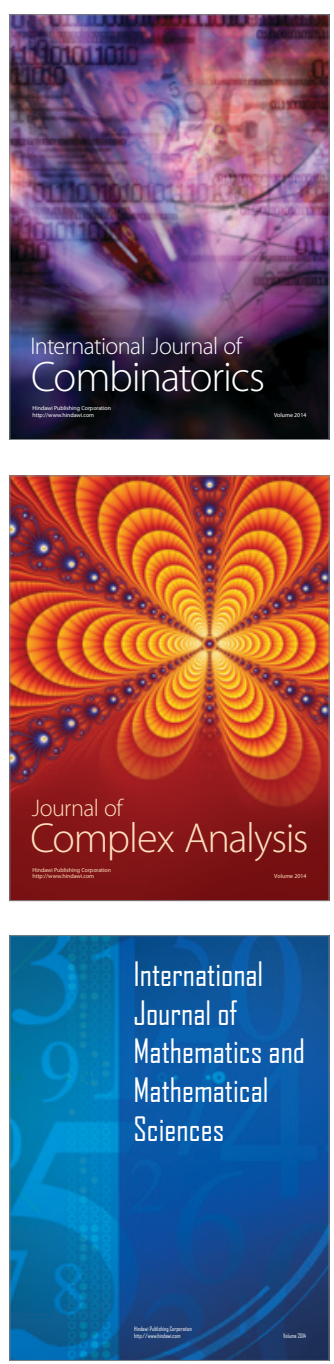
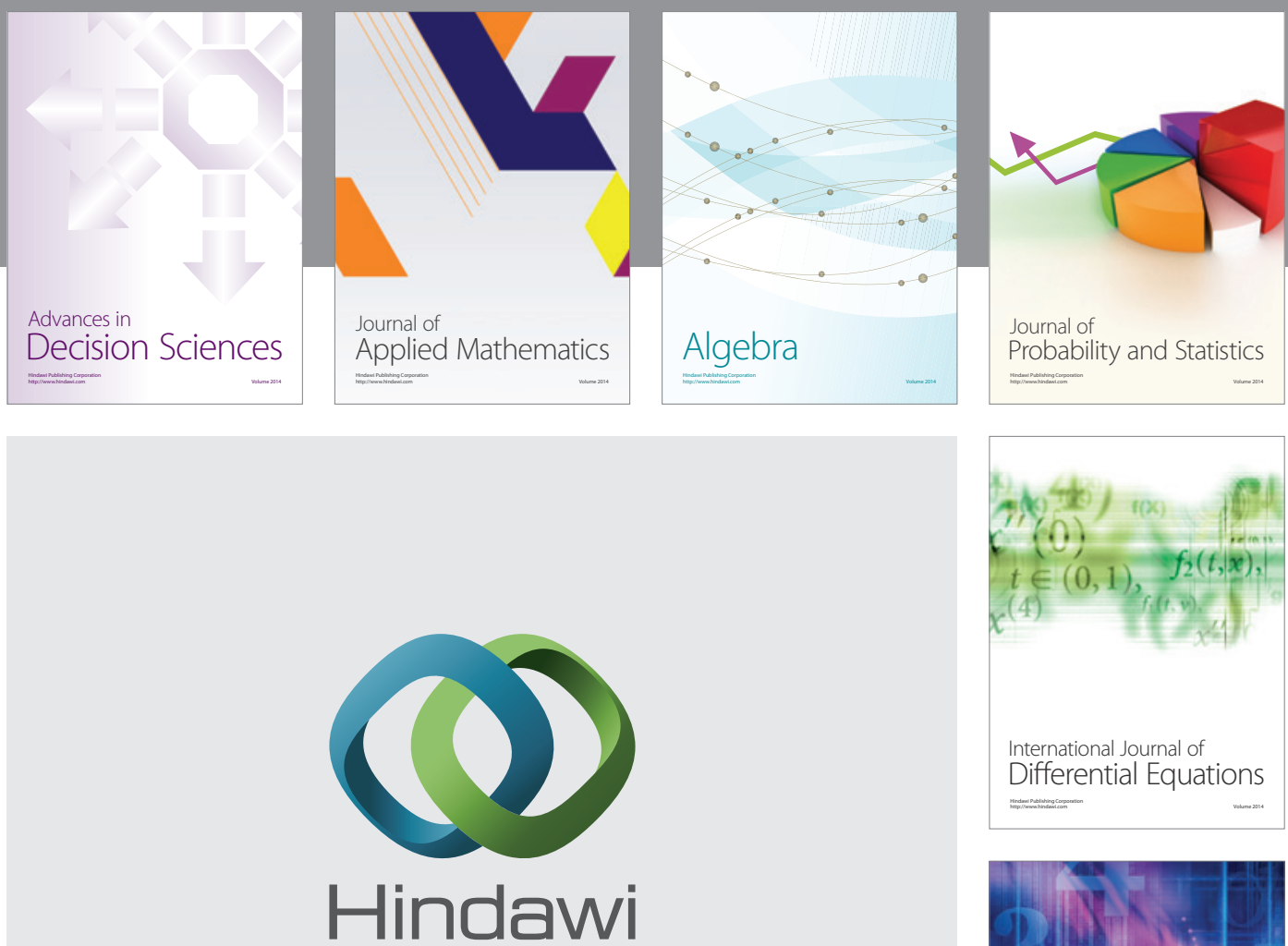

Submit your manuscripts at http://www.hindawi.com
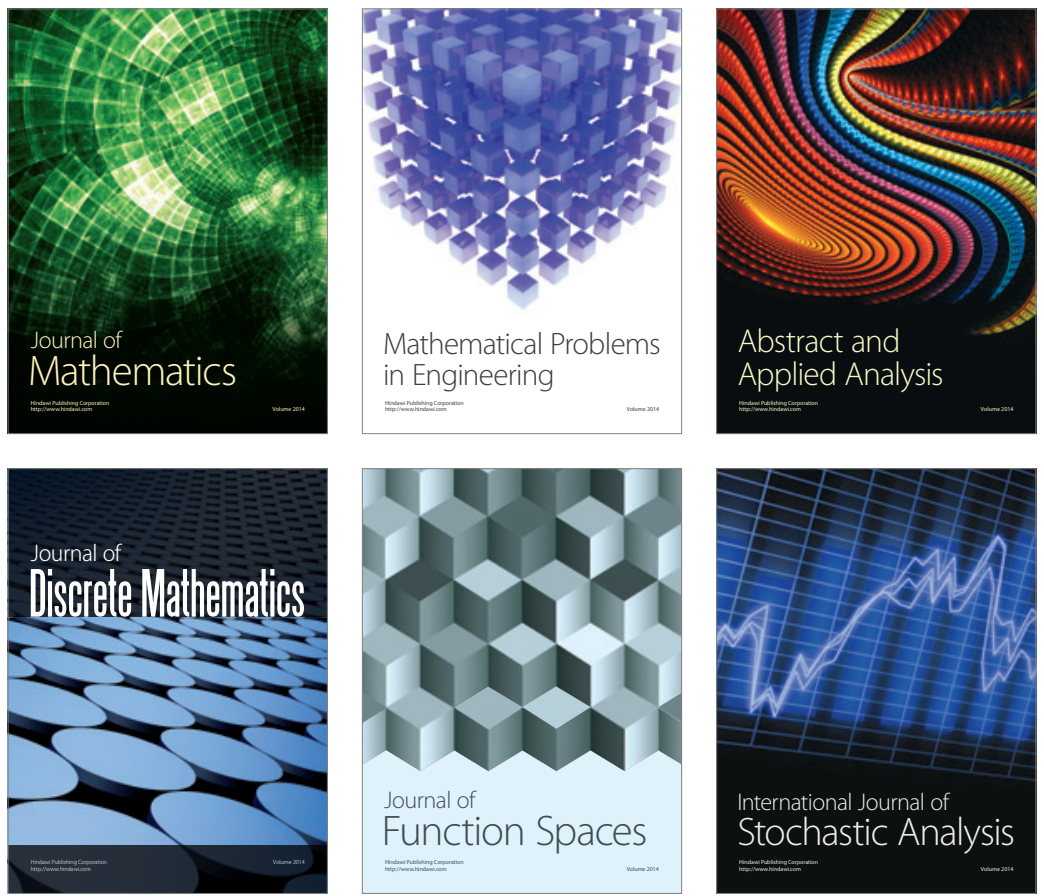

Journal of

Function Spaces

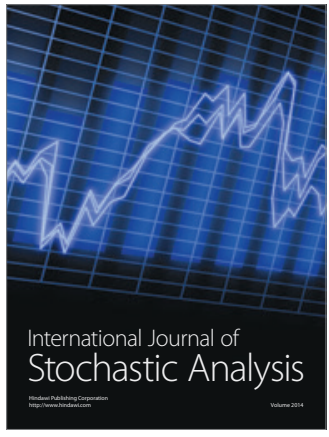

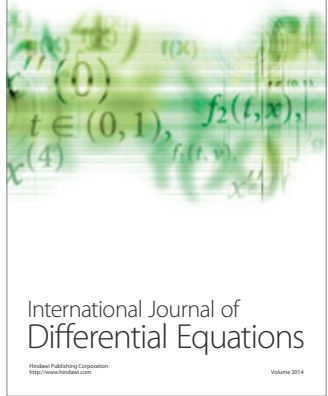
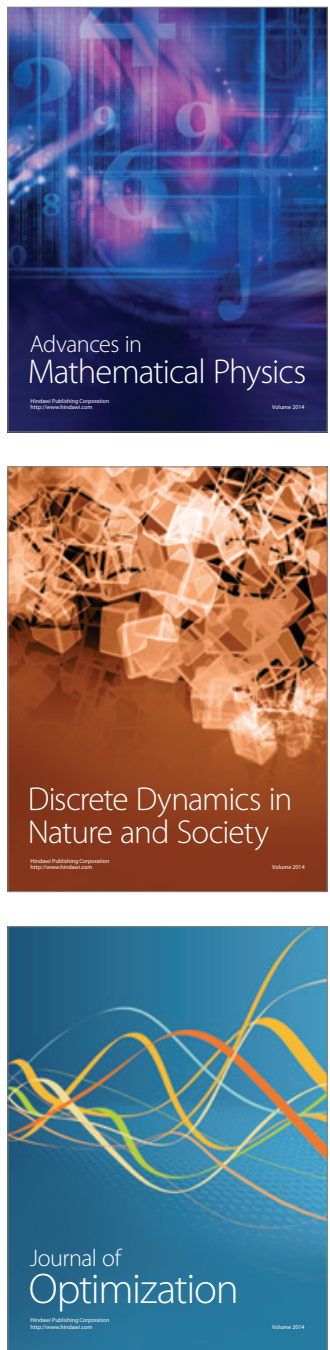